\title{
Kleene Algebras and Semimodules for Energy Problems
}

\author{
Zoltán Ésik ${ }^{1 \star}$, Uli Fahrenberg ${ }^{2}$, Axel Legay ${ }^{2}$, and Karin Quaas $^{3}$ \\ 1 University of Szeged, Hungary \\ 2 Irisa / INRIA Rennes, France \\ 3 Universität Leipzig, Germany
}

\begin{abstract}
With the purpose of unifying a number of approaches to energy problems found in the literature, we introduce generalized energy automata. These are finite automata whose edges are labeled with energy functions that define how energy levels evolve during transitions. Uncovering a close connection between energy problems and reachability and Büchi acceptance for semiring-weighted automata, we show that these generalized energy problems are decidable. We also provide complexity results for important special cases.
\end{abstract}

\section{Introduction}

Energy and resource management problems are important in areas such as embedded systems or autonomous systems. They are concerned with the question whether a given system admits infinite schedules during which (1) certain tasks can be repeatedly accomplished and (2) the system never runs out of energy (or other specified resources). Starting with 8, formal modeling and analysis of such problems has recently attracted some attention [7,9, 13, 16, 20, 27.

As an example, the left part of Fig. 1 1 shows a simple model of an electric car, modeled as a weighted timed automaton [4,5. In the working state $W$, energy is consumed at a rate of 10 energy units per time unit; in the two recharging states $R_{1}, R_{2}$, the battery is charged at a rate of 20 , respectively 10 , energy units per time unit. As the clock $c$ is reset $(c \leftarrow 0)$ when entering state $W$ and has guard $c \geq 1$ on outgoing transitions, we ensure that the car always has to be in state $W$ for at least one time unit. Similarly, the system can only transition back from states $R_{1}, R_{2}$ to $W$ if it has spent at most one time unit in these states.

Passing between states $W$ and $R_{1}$ requires 4 energy units, while transitioning between $W$ and $R_{2}$, and between $R_{2}$ to $R_{1}$, requires 2 energy units. Altogether, this is intended to model the fact that there are two recharge stations available, one close to work but less powerful, and a more powerful one further away. Now

\footnotetext{
* The reserach of this author is supported by the European Union and co-funded by the European Social Fund. Project title: 'Telemedicine-focused research activities on the field of mathematics, informatics and medical sciences'. Project number: TÁMOP-4.2.2.A-11/1/KONV-2012-0073
} 


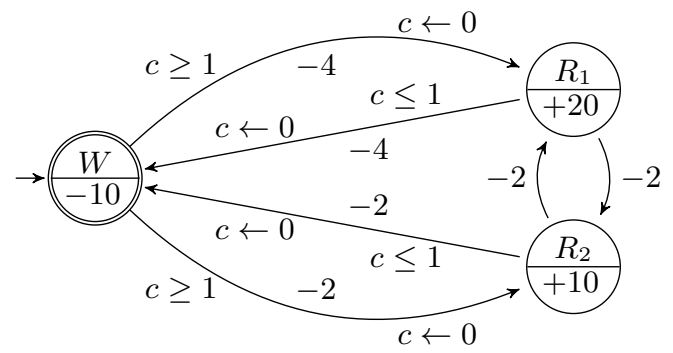

$$
\begin{gathered}
f_{W R}(x)= \begin{cases}\text { undef. } & (x<12) \\
x-12 & (x \geq 12)\end{cases} \\
f_{R W}(x)= \begin{cases}2 x+12 & (x<2) \\
f_{\text {}}+14 & (x \geq 2)\end{cases}
\end{gathered}
$$

Fig. 1. Simple model of an electric car as a weighted timed automaton (left); the corresponding energy automaton (right)

assume that the initial state $W$ is entered with a given initial energy $x_{0}$, then the energy problem of this model is as follows: Does there exist an infinite trace which (1) visits $W$ infinitely often and (2) never has an energy level below 0 ?

This type of energy problems for weighted timed automata is treated in [7, and using a reduction like in [7, our model can be transformed to the energy automaton in the right part of Fig. 1. (The reduction is quite complicated and only works for one-clock timed automata; see [7 for details.) It can be shown that the energy problem for the original automaton is equivalent to the following problem in the energy automaton: Given an initial energy $x_{0}$, and updating the energy according to the transition label whenever taking a transition, does there exist an infinite run which visits $W$ infinitely often? Remark that the energy update on the transition from $R$ to $W$ is rather complex (in the general case of $n$ recharge stations, the definition of $f_{R W}$ can have up to $n$ branches), and that we need to impose a Büchi condition to enforce visiting $W$ infinitely often.

In this paper we propose a generalization of the energy automata of [7] which also encompasses most other approaches to energy problems. Abstracting the properties of the transition update functions in our example, we define a general notion of energy functions which specify how weights change from one system state to another. Noticing that our functional energy automata are semiringweighted automata in the sense of [17, we uncover a close connection between energy problems and reachability and Büchi problems for weighted automata. More precisely, we show that one-dimensional energy problems can be naturally solved using matrix operations in semirings and semimodules [6, 17-19].

For reachability, we use only standard results [17, but for Büchi acceptance we have to extend previous work [18,19] as our semiring is not complete. We thus show that reachability and Büchi acceptance are decidable for energy automata. For the class of piecewise affine energy functions, which generalize the functions of Fig. 1 and are important in applications, they are decidable in exponential time.

Structure of the Paper. We introduce our general model of energy automata in Section 2, In Section 3 we show that the set of energy functions forms a star-continuous Kleene algebra, a fact which allows us to give an elegant char- 
acterization of reachability in energy automata. We also expose a structure of Conway semiring-semimodule pair over energy functions which permits to characterize Büchi acceptance. In Section 4 we use these characterizations to prove that reachability and Büchi acceptance are decidable for energy automata. We also show that this result is applicable to most of the above-mentioned examples and give complexity bounds. To put our results in perspective, we generalize energy automata along several axes in Section 5 and analyze these generalized reachability and Büchi acceptance problems.

Related Work. A simple class of energy automata is the one of integer-weighted automata, where all energy functions are updates of the form $x \mapsto x+k$ for some (positive or negative) integer $k$. Energy problems on these automata, and their extensions to multiple weights (also called vector addition systems with states (VASS)) and games, have been considered e.g. in [8, 10, 14, 20. Our energy automata may hence be considered as a generalization of one-dimensional VASS to arbitrary updates; in the final section of this paper we will also be concerned with multi-dimensional energy automata and games.

Energy problems on timed automata [3] have been considered in [7 [9, 27]. Here timed automata are enriched with integer weights in locations and on transitions (the weighted timed automata of 4,5, cf. Fig. 11), with the semantics that the weight of a delay in a location is computed by multiplying the length of the delay by the location weight. In [8] it is shown that energy problems for oneclock weighted timed automata without updates on transitions (hence only with weights in locations) can be reduced to energy problems on integer-weighted automata with additive updates.

For one-clock weighted timed automata with transition updates, energy problems are shown decidable in [7, using a reduction to energy automata as we use them here. More precisely, each path in the timed automaton in which the clock is not reset is converted to an edge in an energy automaton, labeled with a piecewise affine energy function ( $c f$. Definition 4). Decidability of the energy problem is then shown using ad-hoc arguments, but can easily be inferred from our general results in the present paper.

Also another class of energy problems on weighted timed automata is considered in [7, in which weights during delays are increasing exponentially rather than linearly. These are shown decidable using a reduction to energy automata with piecewise polynomial energy functions; again our present framework applies.

We also remark that semigroups acting on a set, or more generally, semiringsemimodule pairs, have been used to describe the infinitary behavior of automata for a long time, see 6, 26, 30. In this framework, the infinitary product or omega operation is defined on the semiring and takes its values in the semimodule. Another approach is studied e.g. in [25], where the omega operation maps the semiring into itself. It seems to the authors that there is no reasonable definition of an infinitary product or omega operation on energy functions that would again result in an energy function, hence we chose to use the framework of semiringsemimodule pairs. 


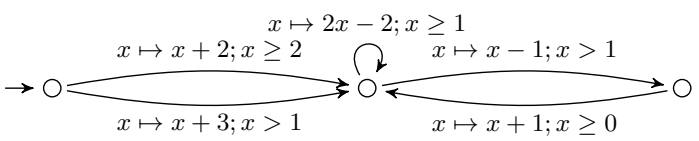

Fig. 2. A simple energy automaton.

\section{Energy Automata}

The transition labels on the energy automata which we consider in the paper, will be functions which model transformations of energy levels between system states. Such transformations have the (natural) properties that below a certain energy level, the transition might be disabled (not enough energy is available to perform the transition), and an increase in input energy always yields at least the same increase in output energy. Thus the following definition.

Definition 1. An energy function is a partial function $f: \mathbb{R}_{\geq 0} \rightarrow \mathbb{R}_{\geq 0}$ which is defined on a closed interval $\left[l_{f}, \infty[\right.$ or on an open interval $] l_{f}, \infty[$, for some lower bound $l_{f} \geq 0$, and such that for all $x_{1} \leq x_{2}$ for which $f$ is defined,

$$
f\left(x_{2}\right) \geq f\left(x_{1}\right)+x_{2}-x_{1}
$$

The class of all energy functions is denoted by $\mathcal{F}$.

Thus energy functions are strictly increasing, and in points where they are differentiable, the derivative is at least 14 The inverse functions to energy functions exist, but are generally not energy functions. Energy functions can be composed, where it is understood that for a composition $g \circ f$ (to be read from right to left), the interval of definition is $\left\{x \in \mathbb{R}_{\geq 0} \mid f(x)\right.$ and $g(f(x))$ defined $\}$. We will generally omit the symbol $\circ$ and write composition simply as $g f$.

Definition 2. An energy automaton $(S, T)$ consists of finite sets $S$ of states and $T \subseteq S \times \mathcal{F} \times S$ of transitions labeled with energy functions.

We show an example of a simple energy automaton in Fig. 2. Here we use inequalities to give the definition intervals of energy functions.

A finite path in an energy automaton is a finite sequence of transitions $\pi=\left(s_{0}, f_{1}, s_{1}\right),\left(s_{1}, f_{2}, s_{2}\right), \ldots,\left(s_{n-1}, f_{n}, s_{n}\right)$. We use $f_{\pi}$ to denote the combined energy function $f_{n} \cdots f_{2} f_{1}$ of such a finite path. We will also use infinite paths, but note that these generally do not allow for combined energy functions.

A global state of an energy automaton is a pair $q=(s, x)$ with $s \in S$ and $x \in \mathbb{R}_{\geq 0}$. A transition between global states is of the form $\left((s, x), f,\left(s^{\prime}, x^{\prime}\right)\right)$ such that $\left(s, f, s^{\prime}\right) \in T$ and $x^{\prime}=f(x)$. A (finite or infinite) run of $(S, T)$ is a path in the graph of global states and transitions.

\footnotetext{
${ }^{4}$ Remark that, in relation to the example in the introduction, the derivative is taken with respect to energy input, not time. Hence the mapping from input to output energy in state $W$ is indeed an energy function in our sense.
} 
We are ready to state the decision problems with which our main concern will lie. As the input to a decision problem must be in some way finitely representable, we will state them for subclasses $\mathcal{F}^{\prime} \subseteq \mathcal{F}$ of computable energy functions; an $\mathcal{F}^{\prime}$ automaton is an energy automaton $(S, T)$ with $T \subseteq S \times \mathcal{F}^{\prime} \times S$.

Problem 1 (Reachability). Given a subset $\mathcal{F}^{\prime}$ of computable functions, an $\mathcal{F}^{\prime}$ automaton $(S, T)$, an initial state $s_{0} \in S$, a set of accepting states $F \subseteq S$, and a computable initial energy $x_{0} \in \mathbb{R}_{\geq 0}$ : does there exist a finite run of $(S, T)$ from $\left(s_{0}, x_{0}\right)$ which ends in a state in $F$ ?

Problem 2 (Büchi acceptance). Given a subset $\mathcal{F}^{\prime}$ of computable functions, an $\mathcal{F}^{\prime}$-automaton $(S, T)$, an initial state $s_{0} \in S$, a set of accepting states $F \subseteq S$, and a computable initial energy $x_{0} \in \mathbb{R}_{\geq 0}$ : does there exist an infinite run of $(S, T)$ from $\left(s_{0}, x_{0}\right)$ which visits $F$ infinitely often?

As customary, a run such as in the statements above is said to be accepting. We let Reach $\mathcal{F}^{\prime}$ denote the function which maps an $\mathcal{F}^{\prime}$-automaton together with an initial state, a set of final states, and an initial energy to the Boolean values ff or tt depending on whether the answer to the concrete reachability problem is negative or positive. Büchi $\mathcal{F}^{\prime}$ denotes the similar mapping for Büchi problems.

The special case of Problem 2 with $F=S$ is the question whether there exists an infinite run in the given energy automaton. This is what is usually referred to as energy problems in the literature; our extension to general Büchi conditions has not been treated before.

\section{The Algebra of Energy Functions}

In this section we develop an algebraic framework of star-continuous Kleene algebra around energy functions which will allow us to solve reachability and Büchi acceptance problems in a generic way. Let $[0, \infty]_{\perp}=\{\perp\} \cup[0, \infty]$ denote the non-negative real numbers together with extra elements $\perp, \infty$, with the standard order on $\mathbb{R}_{\geq 0}$ extended by $\perp<x<\infty$ for all $x \in \mathbb{R}_{\geq 0}$. Also, $\perp+x=$ $\perp-x=\perp$ for all $x \in \mathbb{R}_{\geq 0} \cup\{\infty\}$ and $\infty+x=\infty-x$ for all $x \in \mathbb{R}_{\geq 0}$.

Definition 3. An extended energy function is a mapping $f:[0, \infty]_{\perp} \rightarrow[0, \infty]_{\perp}$, for which $f(\perp)=\perp$ and $f\left(x_{2}\right) \geq f\left(x_{1}\right)+x_{2}-x_{1}$ for all $x_{1} \leq x_{2}$, as in (因). Moreover, $f(\infty)=\infty$, unless $f(x)=\perp$ for all $x \in[0, \infty]_{\perp}$. The class of all extended energy functions is denoted $\mathcal{E}$.

This means, in particular, that $f(x)=\perp$ implies $f\left(x^{\prime}\right)=\perp$ for all $x^{\prime} \leq x$, and $f(x)=\infty$ implies $f\left(x^{\prime}\right)=\infty$ for all $x^{\prime} \geq x$. Hence, except for the extension to $\infty$, these functions are indeed the same as our energy functions from the previous section. Composition of extended energy functions is defined as before, but needs no more special consideration about its definition interval.

We also define an ordering on $\mathcal{E}$, by $f \leq g$ iff $f(x) \leq g(x)$ for all $x \in[0, \infty]_{\perp}$. We will need three special energy functions, $\perp$, id and $\mp$; these are given by $\perp(x)=\perp$, $\operatorname{id}(x)=x$ for $x \in[0, \infty]_{\perp}$, and $\mp(\perp)=\perp, \mp(x)=\infty$ for $x \in[0, \infty]$. 
Lemma 1. With the ordering $\leq, \mathcal{E}$ is a complete lattice with bottom element \pm and top element $\mp$. The supremum on $\mathcal{E}$ is pointwise, i.e. $\left(\sup _{i \in I} f_{i}\right)(x)=$ $\sup _{i \in I} f_{i}(x)$ for any set $I$, all $f_{i} \in \mathcal{E}$ and $x \in[0, \infty]_{\perp}$. Also, $\left(\sup _{i \in I} f_{i}\right) h=$ $\sup _{i \in I}\left(f_{i} h\right)$ for all $h \in \mathcal{E}$.

We denote binary suprema using the symbol $\vee$; hence $f \vee g$, for $f, g \in \mathcal{E}$, is the function $(f \vee g)(x)=\max (f(x), g(x))$.

Lemma 2. ( $\mathcal{E}, \vee, \circ, \pm$, id $)$ is an idempotent semiring with natural order $\leq$.

Recall [17 that $\leq$ being natural refers to the fact that $f \leq g$ iff $f \vee g=g$.

For iterating energy functions, we define a unary star operation on $\mathcal{E}$ by

$$
f^{*}(x)=\left\{\begin{array}{cc}
x & \text { if } f(x) \leq x \\
\infty & \text { if } f(x)>x
\end{array}\right.
$$

Lemma 3. For any $f \in \mathcal{E}$, we have $f^{*} \in \mathcal{E}$. Also, for any $g \in \mathcal{E}$, there exists $f \in \mathcal{E}$ such that $g=f^{*}$ if, and only if, there is $k \in[0, \infty]_{\perp}$ such that $g(x)=x$ for all $x<k, g(x)=\infty$ for all $x>k$, and $g(k)=k$ or $g(k)=\infty$.

By Lemma 1, composition right-distributes over arbitrary suprema in $\mathcal{E}$. The following example shows that a similar left distributivity does not hold in general, hence $\mathcal{E}$ is not a complete semiring the sense of [17. Let $f_{n}, g \in \mathcal{E}$ be defined by $f_{n}(x)=x+1-\frac{1}{n}$ for $x \geq 0, n \in \mathbb{N}_{+}$and $g(x)=x$ for $x \geq 1$. Then $g\left(\sup _{n} f_{n}\right)(0)=$ $g\left(\sup _{n} f_{n}(0)\right)=g(1)=1$, whereas $\left(\sup _{n} g f_{n}\right)(0)=\sup _{n} g\left(f_{n}(0)\right)=\sup _{n} g(1-$ $\left.\frac{1}{n}\right)=\perp$.

The next lemma shows a restricted form of left distributivity which holds only for function powers $f^{n}$. Note that it implies that $f^{*}=\sup _{n} f^{n}$ for all $f \in \mathcal{E}$, which justifies the definition of $f^{*}$ above.

Lemma 4. For any $f, g \in \mathcal{E}, g f^{*}=\sup _{n \in \mathbb{N}}\left(g f^{n}\right)$.

Proposition 1. For any $f, g, h \in \mathcal{E}, g f^{*} h=\sup _{n \in \mathbb{N}}\left(g f^{n} h\right)$. Hence $\mathcal{E}$ is a starcontinuous Kleene algebra [23].

We call a subsemiring $\mathcal{E}^{\prime} \subseteq \mathcal{E}$ a subalgebra if $f^{*} \in \mathcal{E}^{\prime}$ for all $f \in \mathcal{E}^{\prime}$.

It is known 6, 15, 17, 24 that when $S$ is a star-continuous Kleene algebra, then so is any matrix semiring $S^{n \times n}$, for all $n \geq 1$, with the usual sum and product operations. The natural order on $S^{n \times n}$ is pointwise, so that for all $n \times n$ matrices $A, B$ over $S, A \leq B$ iff $A_{i, j} \leq B_{i, j}$ for all $i, j$. Now a star-continuous Kleene algebra is also a Conway semiring, hence the Conway identities

$$
(g \vee f)^{*}=\left(g^{*} f\right)^{*} g^{*} \quad \text { and } \quad(g f)^{*}=g(f g)^{*} f \vee \text { id }
$$

are satisfied for all $f, g \in \mathcal{E}$. Also, this implies that the matrix semiring $\mathcal{E}^{n \times n}$ is again a Conway semiring, for any $n \geq 1$, with the star operation defined inductively for a matrix

$$
M=\left[\begin{array}{ll}
a & b \\
c & d
\end{array}\right] \in \mathcal{E}^{n \times n},
$$


where $a$ is $k \times k$ and $d$ is $m \times m$ with $k+m=n$, by

$$
M^{*}=\left[\begin{array}{cc}
\left(a \vee b d^{*} c\right)^{*} & \left(a \vee b d^{*} c\right)^{*} b d^{*} \\
\left(d \vee c a^{*} b\right)^{*} c a^{*} & \left(d \vee c a^{*} b\right)^{*}
\end{array}\right] \in \mathcal{E}^{n \times n}
$$

The definition of $M^{*}$ does not depend on how $M$ is split into parts, and starcontinuity implies that for all matrices $M, N, O$,

$$
N M^{*} O=\sup _{n \in \mathbb{N}}\left(N M^{n} O\right)
$$

Note again that this implies that $M^{*}=\sup _{n} M^{n}$ for all matrices $M$. In a sense, this gives another, inductive definition of the star operation on the matrix semiring; the important property of star-continuous Kleene algebras is, then, that this inductive definition and the one in (3) give rise to the same operation.

We introduce a semimodule $\mathcal{V}$ over $\mathcal{E}$. Let $\mathcal{B}=\{\mathrm{ff}, \mathrm{tt}\}$ be the Boolean algebra, with order $\mathrm{ff}<\mathrm{tt}$, and $\mathcal{V}=\left\{u:[0, \infty]_{\perp} \rightarrow \mathcal{B} \mid u(\perp)=\mathrm{ff}, x_{1} \leq x_{2} \Rightarrow u\left(x_{1}\right) \leq\right.$ $\left.u\left(x_{2}\right)\right\}$. Identifying $\mathrm{ff}$ with $\perp$ and $\mathrm{tt}$ with $\infty$, we have an embedding of $\mathcal{V}$ into $\mathcal{E}$; note that $\perp, \mp \in \mathcal{V}$.

Lemma 5. With action $(u, f) \mapsto u f: \mathcal{V} \times \mathcal{E} \rightarrow \mathcal{V}, \mathcal{V}$ is a right $\mathcal{E}$-semimodule [19]. Moreover, $\left(\sup _{i \in I} u_{i}\right) f=\sup _{i \in I}\left(u_{i} f\right)$ for any set $I$, all $u_{i} \in \mathcal{V}$ and $f \in \mathcal{E}$, and $u f^{*}=\sup _{n \in \mathbb{N}} u f^{n}$ for all $u \in \mathcal{V}$.

So like the situation for $\mathcal{E}(c f$. Lemmas 1 and 4$)$, the action of $\mathcal{E}$ on $\mathcal{V}$ rightdistributes over arbitrary suprema and left-distributes over function powers.

We define an infinitary product operation $\mathcal{E}^{\omega} \rightarrow \mathcal{V}$. Let $f_{0}, f_{1}, \ldots$ be an infinite sequence of energy functions and $x_{0} \in[0, \infty]_{\perp}$, and put $x_{n+1}=f_{n}\left(x_{n}\right)$ for $n \in \mathbb{N}$. Then we define

$$
\left(\prod_{i=0}^{\infty} f_{i}\right)\left(x_{0}\right)= \begin{cases}\mathrm{ff} & \text { if } \exists n \in \mathbb{N}: x_{n}=\perp \\ \mathrm{tt} & \text { if } \forall n \in \mathbb{N}: x_{n} \neq \perp\end{cases}
$$

Note that this product is order-preserving. By the next lemma, it is a conservative extension of the finite product. As $\mathcal{E}$ is not a complete semiring, it follows that $(\mathcal{E}, \mathcal{V})$ is not a complete semiring-semimodule pair in the sense of [19].

Lemma 6. For all $f_{0}, f_{1}, \ldots \in \mathcal{E},\left(\prod_{i=1}^{\infty} f_{i}\right) f_{0}=\prod_{i=0}^{\infty} f_{i}$. For all indices $0=$ $n_{0} \leq n_{1} \leq \ldots, \prod_{i=0}^{\infty} f_{i}=\prod_{i=0}^{\infty}\left(f_{n_{i+1}-1} \cdots f_{n_{i}}\right)$.

To deal with infinite iterations of energy functions, we define a unary omega operation $\mathcal{E} \rightarrow \mathcal{V}$ by

$$
f^{\omega}(x)= \begin{cases}\mathrm{ff} & \text { if } x=\perp \text { or } f(x)<x \\ \mathrm{tt} & \text { if } x \neq \perp \text { and } f(x) \geq x\end{cases}
$$

Note that $f^{\omega}=\prod_{i=0}^{\infty} f$ for all $f \in \mathcal{E}$.

Proposition 2. $(\mathcal{E}, \mathcal{V})$ is a Conway semiring-semimodule pair. 
Recall [6] that this means that additionally to the identities (1),

$$
(g f)^{\omega}=(f g)^{\omega} f \quad \text { and } \quad(f \vee g)^{\omega}=f^{\omega}\left(g f^{*}\right)^{*} \vee\left(g f^{*}\right)^{\omega}
$$

for all $f, g \in \mathcal{E}$. Like for Conway semirings, it implies that the pair $\left(\mathcal{E}^{n \times n}, \mathcal{V}^{n}\right)$ is again a Conway semiring-semimodule pair, for any $n \geq 1$, with the action of $\mathcal{E}^{n \times n}$ on $\mathcal{V}^{n}$ similar to matrix-vector multiplication using the action of $\mathcal{E}$ on $\mathcal{V}$, and the omega operation $\mathcal{E}^{n \times n} \rightarrow \mathcal{V}^{n}$ given inductively as follows: for $M \in \mathcal{E}^{n \times n}$ with blocks as in (2), define

$$
\begin{array}{cl}
M^{\omega}=\left[\left(a \vee b d^{*} c\right)^{\omega} \vee d^{\omega} c\left(a \vee b d^{*} c\right)^{*}\right. & \left.\left(d \vee c a^{*} b\right)^{\omega} \vee a^{\omega} b\left(d \vee c a^{*} b\right)^{*}\right], \\
M^{\omega_{k}}=\left[\left(a \vee b d^{*} c\right)^{\omega}\right. & \left.\left(a \vee b d^{*} c\right)^{\omega} b d^{*}\right] .
\end{array}
$$

The definition of $M^{\omega}$ does not depend on how $M$ is split into parts, but the one of $M^{\omega_{k}}$ does (recall that $a$ is a $k \times k$ matrix). It can be shown [6] that (5), and also (3), follow directly from certain general properties of fixed point operations.

\section{Decidability}

We are now ready to apply the Kleene algebra framework to reachability and Büchi acceptance for energy automata. We first show that it is sufficient to consider energy automata $(S, T)$ with precisely one transition $\left(s, f, s^{\prime}\right) \in T$ for each pair of states $s, s^{\prime} \in S$. This will allow us to consider $T$ as a matrix $S \times S \rightarrow \mathcal{E}$ (as is standard in weighted-automata theory [17]).

Lemma 7. Let $\mathcal{E}^{\prime} \subseteq \mathcal{E}$ be a subalgebra and $(S, T)$ an $\mathcal{E}^{\prime}$-automaton. There exists an $\mathcal{E}^{\prime}$-automaton $\left(S, T^{\prime}\right)$ for which $\operatorname{Reach}_{\mathcal{E}^{\prime}}(S, T)=\operatorname{Reach}_{\mathcal{E}^{\prime}}\left(S, T^{\prime}\right)$ and $\operatorname{Büchi}_{\mathcal{E}^{\prime}}(S, T)=\operatorname{Büchi}_{\mathcal{E}^{\prime}}\left(S, T^{\prime}\right)$, and in which there is precisely one transition $\left(s, f, s^{\prime}\right) \in T^{\prime}$ for all $s, s^{\prime} \in S$.

Hence we may, without loss of generality, view the transitions $T$ of an energy automaton as a matrix $T: S \times S \rightarrow \mathcal{E}$. We can also let $S=\{1, \ldots, n\}$ and assume that the set of accepting states is $F=\{1, \ldots, k\}$ for $k \leq n$. Further, we can represent an initial state $s_{0} \in S$ by the $s_{0}$ th unit (column) vector $I^{s_{0}} \in\{ \pm \text {, id }\}^{n}$, defined by $I_{i}^{s_{0}}=$ id iff $i=s_{0}$, and $F$ by the (column) vector $F^{\leq k} \in\{ \pm \text {, id }\}^{n}$ given by $F_{i}^{\leq k}=$ id iff $i \leq k$. Note that $T \in \mathcal{E}^{n \times n}$ is an $n \times n$-matrix of energy functions; as composition of energy functions is written right-to-left, $T_{i j} \in \mathcal{E}$ is the function on the transition from $s_{j}$ to $s_{i}$.

Theorem 1. Let $\mathcal{E}^{\prime} \subseteq \mathcal{E}$ be a subalgebra. For any $\mathcal{E}^{\prime}$-automaton $(S, T)$ with $S=\{1, \ldots, n\}, F=\{1, \ldots, k\}, k \leq n, s_{0} \leq n$, and $x_{0} \in \mathbb{R}_{\geq 0}$, we have $\operatorname{Reach}_{\mathcal{E}^{\prime}}(S, T)\left(F, s_{0}, x_{0}\right)=\mathbf{t t}$ if, and only if, ${ }_{\mathrm{t}} F^{\leq k} T^{*} I^{s_{0}}\left(x_{0}\right) \neq \perp$.

Proof. Here ${ }_{\mathrm{t}} F^{\leq k}$ denotes the transpose of $F^{\leq k}$. By (4), we have ${ }_{\mathrm{t}} F^{\leq k} T^{*} I^{s_{0}}=$ $\sup _{n}\left({ }_{\mathrm{t}} F^{\leq k} T^{n} I^{s_{0}}\right)$, so that ${ }_{\mathrm{t}} F^{\leq k} T^{*} I^{s_{0}}\left(x_{0}\right) \neq \perp$ iff ${ }_{\mathrm{t}} F^{\leq k} T^{n} I^{s_{0}}\left(x_{0}\right) \neq \perp$ for some $n \in \mathbb{N}$, i.e. iff there is a finite run from $\left(s_{0}, x_{0}\right)$ which ends in a state in $F$. 


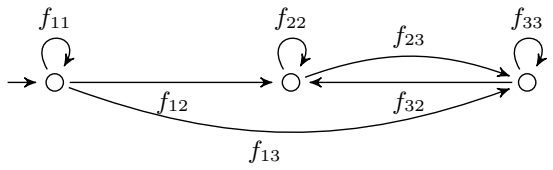

$$
\begin{aligned}
& f_{11}(x)=x ; \quad x \geq 0 \\
& f_{12}(x)=\infty ; \quad x>1 \\
& f_{23}(x)= \begin{cases}x-1 ; & 1<x \leq 2 \\
\infty ; & x>2\end{cases} \\
& f_{33}(x)= \begin{cases}x ; & x \leq 1 \\
\infty ; & x>1\end{cases} \\
& f_{22}(x)= \begin{cases}x ; & x \leq 2 \\
\infty ; & x>2\end{cases} \\
& f_{32}(x)= \begin{cases}x+1 ; & x \leq 1 \\
\infty ; & x>1\end{cases} \\
& f_{13}(x)=\infty ; \quad x>1
\end{aligned}
$$

Fig. 3. The closure of the automaton from Fig. 2 ,

Referring back to the example automaton $(S, T)$ from Fig. 2, we display in Fig. 3 the automaton with transition matrix $T^{*}$.

Theorem 2. Let $\mathcal{E}^{\prime} \subseteq \mathcal{E}$ be a subalgebra. For any $\mathcal{E}^{\prime}$-automaton $(S, T)$ with $S=\{1, \ldots, n\}, F=\{1, \ldots, k\}, k \leq n, s_{0} \leq n$, and $x_{0} \in \mathbb{R}_{\geq 0}$, we have $\operatorname{Büchi}_{\mathcal{E}^{\prime}}(S, T)\left(F, s_{0}, x_{0}\right)=T^{\omega_{k}} I^{s_{0}}\left(x_{0}\right)$.

Proof. This is a standard result for complete semiring-semimodule pairs, $c f$. [19]. Now $(\mathcal{E}, \mathcal{V})$ is not complete, but the properties developed in the previous section allow us to show the result nevertheless. We need to see that for all $M \in \mathcal{E}^{n \times n}$ and $1 \leq i \leq n$,

$$
\left(M^{\omega}\right)_{i}=\sup \left\{\cdots M_{k_{3}, k_{2}} M_{k_{2}, k_{1}} M_{k_{1}, i}: 1 \leq k_{1}, k_{2}, \ldots \leq n\right\},
$$

which we shall deduce inductively from (5).

Let $a \in \mathcal{E}^{\ell \times \ell}, d \in \mathcal{E}^{m \times m}$, for $\ell+m=n$, and let $i \in\{1, \ldots, \ell\}$. Then the $i$ th component of $M^{\omega}$ is the $i$ th component of $\left(a \vee b d^{*} c\right)^{\omega} \vee d^{\omega} c\left(a \vee b d^{*} c\right)^{*}$. By induction hypothesis, the $i$ th component of $\left(a \vee b d^{*} c\right)^{\omega}$ is the supremum of all infinite products $\left(\cdots M_{k_{2}, k_{1}} M_{k_{1}, i}\right)$ such that $1 \leq k_{j} \leq m$ for an infinite number of indices $j$, and similarly, the $i$ th component of $d^{\omega} c\left(a \vee b d^{*} c\right)^{*}$ is the supremum of all infinite products $\left(\cdots M_{k_{2}, k_{1}} M_{k_{1}, i}\right)$ such that $1 \leq k_{j} \leq m$ for a finite number of indices $j$. Thus, the $i$ th component of $\left(a \vee b d^{*} c\right)^{\omega} \vee d^{\omega} c\left(a \vee b d^{*} c\right)^{*}$ is the supremum of all infinite products $\left(\cdots M_{k_{2}, k_{1}} M_{k_{1}, i}\right)$.

We remark that our decision algorithms are static in the sense that the matrix expressions can be pre-computed and then re-used to decide reachability and Büchi acceptance for different values $x_{0}$ of initial energies.

Using elementary reasoning on infinite paths, we can provide an alternative characterization of Büchi acceptance which does not use the omega operations:

Theorem 3. Let $\mathcal{E}^{\prime} \subseteq \mathcal{E}$ be a subalgebra. For any $\mathcal{E}^{\prime}$-automaton $(S, T)$ with $S=\{1, \ldots, n\}, F=\{1, \ldots, k\}, k \leq n, s_{0} \leq n$, and $x_{0} \in \mathbb{R}_{\geq 0}$, we have $\operatorname{Büchi}_{\mathcal{E}^{\prime}}(S, T)\left(F, s_{0}, x_{0}\right)=\mathbb{t t}$ if, and only if, there exists $j \leq k$ for which

$$
{ }_{\mathrm{t}} I^{j} T T^{*} I^{j}{ }_{\mathrm{t}} I^{j} T^{*} I^{s_{0}}\left(x_{0}\right) \geq_{\mathrm{t}} I^{j} T^{*} I^{s_{0}}\left(x_{0}\right) \neq \perp .
$$


Corollary 1. For subalgebras $\mathcal{E}^{\prime} \subseteq \mathcal{E}$ of computable functions in which it is decidable for each $f \in \mathcal{E}^{\prime}$ whether $f(x) \leq x$, Problems 1 and 0 are decidable. For an energy automaton with $n$ states and $m$ transitions, the decision procedures use $O\left(m+n^{3}\right)$, respectively $O\left(m+n^{4}\right)$, algebra operations.

Proof. Maxima and compositions of computable functions are again computable, and if it is decidable for each $f \in \mathcal{E}^{\prime}$ whether $f(x) \leq x$, then also $f^{*}$ is computable for each $f \in \mathcal{E}^{\prime}$. Hence all matrix operations used in Lemma 7 and Theorems 1 and 3 are computable. The number of operations necessary in the construction in the proof of Lemma 7 is $O(m)$, and, using e.g. the Floyd-Warshall algorithm to compute $T^{*}, O\left(n^{3}\right)$ operations are necessary to compute ${ }_{\mathrm{t}} I \leq k T^{*} I^{s_{0}}$.

We proceed to identify two important subclasses of computable energy functions, which cover most of the related work mentioned in the introduction, and to give complexity results on their reachability and Büchi acceptance problems.

The integer update functions in $\mathcal{E}$ are the functions $f_{k}$, for $k \in \mathbb{Z}$, given by

$$
f_{k}(x)=\left\{\begin{array}{cl}
x+k & \text { if } x \geq \max (0,-k), \\
\perp & \text { otherwise }
\end{array}\right.
$$

together with $f_{\infty}:=\mp$. These are the update functions usually considered in integer-weighted automata and VASS $\left[8,10,14,20\right.$. We have $f_{\ell} f_{k}=f_{k+\ell}$ and $f_{k} \vee f_{\ell}=f_{\max (k, \ell)}$, and $f_{k}^{*}=f_{0}$ for $k \leq 0$ and $f_{k}^{*}=f_{\infty}$ for $k>0$, whence the class $\mathcal{E}_{\text {int }}$ of integer update functions forms a subalgebra of $\mathcal{E}$. A function $f_{k} \in \mathcal{E}_{\text {int }}$ can be represented by the integer $k$, and algebra operations can then be performed in constant time. Hence Corollary 1 implies the following result.

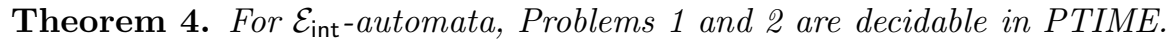

Next we turn our attention to piecewise affine functions as used in Fig. 1

Definition 4. A function $f \in \mathcal{E}$ is said to be (rational) piecewise affine if there exist $x_{0}<x_{1}<\cdots<x_{k} \in \mathbb{Q}$ such that $f(x) \neq \perp$ iff $x \geq x_{0}$ or $x>x_{0}$,

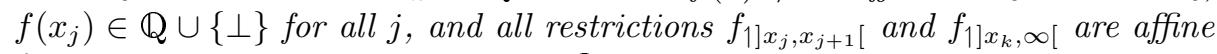
functions $x \mapsto a_{j} x+b_{j}$ with $a_{j}, b_{j} \in \mathbb{Q}, a_{j} \geq 1$.

Note that the definition does not make any assertion about continuity at the $x_{j}$, but (圈) implies that $\lim _{x \nearrow x_{j}} f(x) \leq f\left(x_{j}\right) \leq \lim _{x \searrow x_{j}} f(x)$. A piecewise affine function as above can be represented by its break points $x_{0}, \ldots, x_{k}$, the values $f\left(x_{0}\right), \ldots, f\left(x_{k}\right)$, and the numbers $a_{0}, b_{0}, \ldots, a_{k}, b_{k}$. These functions arise in the reduction used in [7] to show decidability of energy problems for one-clock timed automata with transition updates. The notion of integer piecewise affine functions is defined similarly, with all occurrences of $\mathbb{Q}$ above replaced by $\mathbb{Z}$. Fig. 4 shows an example of a piecewise affine function.

The class $\mathcal{E}_{\mathrm{pw}}$ of piecewise affine energy functions forms a subsemiring of $\mathcal{E}$ : if $f, g \in \mathcal{E}_{\mathrm{pw}}$ with break points $x_{0}, \ldots, x_{k}, y_{0}, \ldots, y_{\ell}$, respectively, then $f \vee g$ is piecewise affine with break points a subset of $\left\{x_{0}, \ldots, x_{k}, y_{0}, \ldots, y_{\ell}\right\}$, and $g f$ is piecewise affine with break points a subset of $\left\{x_{0}, \ldots, x_{k}, f^{-1}\left(y_{0}\right), \ldots, f^{-1}\left(y_{\ell}\right)\right\}$. 


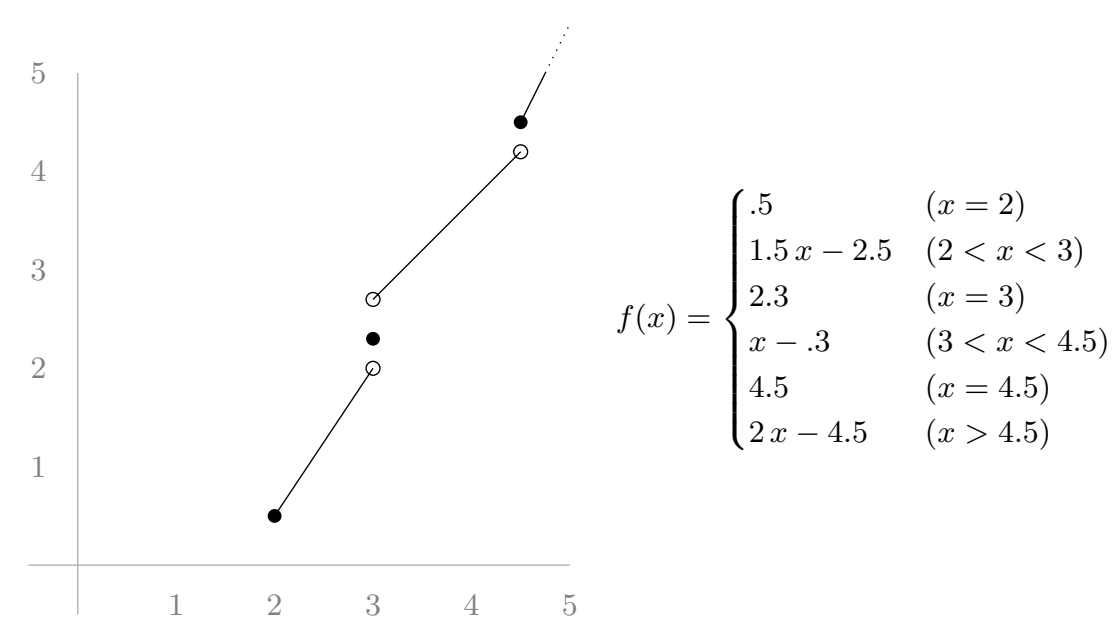

Fig. 4. A piecewise affine energy function

Let, for any $k \in \mathbb{Q}, g_{k}^{-}, g_{k}^{+}:[0, \infty]_{\perp} \rightarrow[0, \infty]_{\perp}$ be the functions defined by

$$
g_{k}^{-}(x)=\left\{\begin{array}{cc}
x & \text { for } x<k, \\
\infty & \text { for } x \geq k,
\end{array} \quad g_{k}^{+}(x)=\left\{\begin{array}{cc}
x & \text { for } x \leq k \\
\infty & \text { for } x>k
\end{array}\right.\right.
$$

By Lemma 3 (and noticing that for all $f \in \mathcal{E}_{\mathrm{pw}}, \sup \{x \mid f(x) \leq x\}$ is rational), $\mathcal{E}_{\text {pw }}$ completed with the functions $g_{k}^{-}, g_{k}^{+}$forms a subalgebra of $\mathcal{E}$.

Remark that, unlike $\mathcal{E}_{\mathrm{pw}}$, the class $\mathcal{E}_{\mathrm{pwi}}$ of integer piecewise affine functions does not form a subsemiring of $\mathcal{E}$, as composites of $\mathcal{E}_{\text {pwi-functions are not neces- }}$ sarily integer piecewise affine. As an example, for the functions $f, g \in \mathcal{E}_{\text {pwi }}$ given by

$$
f(x)=2 x, \quad g(x)= \begin{cases}x+1 ; & x<3, \\ x+2 ; & x \geq 3,\end{cases}
$$

we have

$$
g(f(x))= \begin{cases}2 x+1 ; & x<1.5 \\ 2 x+2 ; & x \geq 1.5\end{cases}
$$

which is not integer piecewise affine. Similarly, the class of rational affine functions $x \mapsto a x+b$ (without break points) is not closed under maximum, and $\mathcal{E}_{\mathrm{pw}}$ is the semiring generated by rational affine functions.

Theorem 5. For $\mathcal{E}_{\mathrm{pw}}$-automata, Problems 1 and 0 are decidable in EXPTIME.

Proof. We need to show that it is decidable for each $f \in \mathcal{E}_{\mathrm{pw}}$ whether $f(x) \leq x$. Let thus $f$ be a piecewise affine function, with representation $\left(x_{0}, \ldots, x_{k}, f\left(x_{0}\right)\right.$, $\left.\ldots, f\left(x_{k}\right), a_{0}, \ldots, a_{k}, b_{0}, \ldots, b_{k}\right)$. If $x<x_{0}$, then $f(x)=\perp \leq x$. If $x=x_{j}$ for some $j$, we can simply compare $x_{j}$ with $f\left(x_{j}\right)$. 


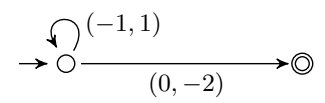

Fig. 5. A simple two-dimensional VASS

Assume now that $x \in] x_{j}, x_{j+1}\left[\right.$ for some $j$. If $a_{j} x_{j}+b_{j} \leq x_{j}$ and $a_{j} x_{j+1}+b_{j} \leq$ $x_{j+1}$, then also $f(x) \leq x$ by (因). Likewise, if $a_{j} x_{j}+b_{j}>x_{j}$ and $a_{j} x_{j+1}+b_{j}>$ $x_{j+1}$, then also $f(x)>x$. The case $a_{j} x_{j}+b_{j}>x_{j}, a_{j} x_{j+1}+b_{j} \leq x_{j+1}$ cannot occur because of (図), and if $a_{j} x_{j}+b_{j} \leq x_{j}$ and $a_{j} x_{j+1}+b_{j}>x_{j+1}$, then $a_{j}>1$, and $f(x) \leq x$ iff $x \leq \frac{b_{j}}{1-a_{j}}$.

For the case $x \in] x_{k}, \infty\left[\right.$, the arguments are similar: if $a_{k} x_{k}+b_{k}>x_{k}$, then also $f(x)>x$; if $a_{k} x_{k}+b_{k} \leq x_{k}$ and $a_{k}=1$, then also $f(x) \leq x$, and if $a_{k}>1$ in this case, then $f(x) \leq x$ iff $x \leq \frac{b_{k}}{1-a_{k}}$.

Using Corollary 1, we have hence shown decidability. For the complexity claim, we note that all algebra operations in $\mathcal{E}_{\mathrm{pw}}$ can be performed in time linear in the size of the representations of the involved functions. However, the maximum and composition operations may double the size of the representations, hence our procedure may take time $O\left(2^{m+n^{3}} p\right)$ for reachability, and $O\left(2^{m+n^{4}} p\right)$ for Büchi acceptance, for an $\mathcal{E}_{\mathrm{pw}}$-automaton with $n$ states, $m$ transitions, and energy functions of representation length at most $p$.

In the setting of $\mathcal{E}_{\mathrm{pw}}$-automata and their application to one-clock weighted timed automata with transition updates, our Theorem 3 is a generalization of [7, Lemmas 24, 25]. Complexity of the decision procedure was left open in [7; as the conversion of a one-clock weighted timed automaton to an $\mathcal{E}_{\mathrm{pw}}$-automaton incurs an exponential blowup, we now see that their procedure is doubly-exponential.

Considerations similar to the above show that also the setting of piecewise polynomial energy functions allows an application of Theorem 3 to show energy problems on the exponentially weighted timed automata from [7] decidable.

\section{Multi-dimensional Energy Automata and Games}

Next we turn our attention to several variants of energy automata. We will generally stick to the set $\mathcal{E}_{\text {pwi }}$ of integer piecewise affine energy functions; the fact that $\mathcal{E}_{\text {pwi }}$ is not a subsemiring of $\mathcal{E}$ will not bother us here.

An $n$-dimensional integer piecewise affine energy automaton, or $\mathcal{E}_{\mathrm{pwi}}^{n}$-automaton for short, $(S, T)$, for $n \in \mathbb{N}_{+}$, consists of finite sets $S$ of states and $T \subseteq S \times \mathcal{E}_{\text {pwi }}^{n} \times S$ of transitions. A global state in such an automaton is a pair $(s, \boldsymbol{x}) \in S \times \mathbb{N}^{n}$, and transitions are of the form $(s, \boldsymbol{x}) \stackrel{f}{\rightarrow}\left(s^{\prime}, \boldsymbol{x}^{\prime}\right)$ such that $\left(s, \boldsymbol{f}, s^{\prime}\right) \in T$ and $\boldsymbol{x}^{\prime}(i)=\boldsymbol{f}(i)(\boldsymbol{x}(i))$ for each $i \in\{1, \ldots, n\}$.

For reachability in $\mathcal{E}_{\text {pwi }}^{n}$-automata (with $n \geq 2$ ), our algebraic results do not apply. To see this, we refer to the reachability problem in Fig. 5: with initial energy $(1,1)$, the loop needs to be taken precisely once, but with initial energy 
$(2,0)$, one needs to loop twice. Hence there is no static algorithm which can decide reachability for this VASS.

However, we remark that $\mathcal{E}_{\text {pwi }}^{n}$-automata are well-structured transition systems 22], with ordering on global states defined by $(s, \boldsymbol{x}) \preceq\left(s^{\prime}, \boldsymbol{x}^{\prime}\right)$ iff $s=s^{\prime}$ and $\boldsymbol{x}(i) \leq \boldsymbol{x}^{\prime}(i)$ for each $i=1, \ldots, n$ (here we also have to assume $x_{0} \in \mathbb{N}$ ). Also, the reachability problem for energy automata is a control state reachability problem in the sense of 2 . Decidability of the reachability problem for $\mathcal{E}_{\text {pwi }}^{n}$-automata thus follows from the decidability of the control state reachability problem for well-structured transition systems [2]. Note that Büchi acceptance is not generally decidable for well-structured transition systems (it is undecidable for lossy counter machines [29]), so our reduction proof does not imply a similar result for Büchi acceptance.

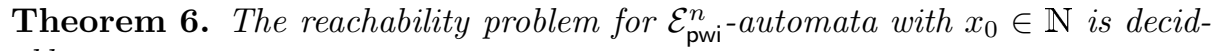
able.

Next we show that if the requirement (図) on energy functions, that $f\left(x_{2}\right) \geq$ $f\left(x_{1}\right)+x_{2}-x_{1}$ for each $x_{1} \leq x_{2}$, is lifted, then reachability becomes undecidable from dimension 4 . We call such functions flat energy functions; remark that we still require them to be strictly increasing, but the derivative, where it exists, may be less than 1 . The class of all flat energy functions is denoted $\overline{\mathcal{E}}$ and its restrictions by $\overline{\mathcal{E}}_{\mathrm{pw}}, \overline{\mathcal{E}}_{\mathrm{pwi}}$.

Theorem 7. The reachability problem for $\overline{\mathcal{E}}_{\mathrm{pw}}^{4}$-automata is undecidable.

Next we extend our energy automata formalism to (turn based) reachability games. Let $(S, T)$ be an $n$-dimensional energy automaton such that $S=S_{A} \cup S_{B}$ forms a partition of $S$ and $T \subseteq\left(S_{A} \times \mathcal{E}_{\text {pwi }}^{n} \times S_{B}\right) \cup\left(S_{B} \times \mathcal{E}_{\text {pwi }}^{n} \times S_{A}\right)$. Then $\left(S, S_{A}, S_{B}, T\right)$ induces an $n$-dimensional energy game $G$. The intuition of the reachability game is that the two players $A$ and $B$ take turns to move along the game graph $(S, T)$, updating energy values at each turn. The goal of player $A$ is to reach a state in $F$, the goal of player $B$ is to prevent this from happening.

The reachability game is a coverability game in the sense of [28. In general, the reachability game on well-structured transition systems is undecidable [1]. Indeed, the games on VASS considered in [10] are a special case of reachability games on energy automata with integer update functions; their undecidability is shown in [1]10]. It is hence clear that it is undecidable whether player $A$ wins the reachability game in 2 -dimensional $\mathcal{E}_{\text {int-automata. As a corollary, we can show }}$ that for flat energy functions, already one-dimensional reachability games are undecidable.

Theorem 8. Whether player $A$ wins the reachability game in $\mathcal{E}_{\text {int }}^{2}$-automata is undecidable.

Theorem 9. It is undecidable for $\overline{\mathcal{E}}_{\mathrm{pw}}$-automata whether player $A$ wins the reachability game. 
Proof (sketch). The proof is by reduction from reachability games on 2-dimensional $\mathcal{E}_{\text {int }}$-automata to reachability games on 1-dimensional $\overline{\mathcal{E}}_{\text {pwi-automata. The }}$ intuition is that the new energy variable $x$ encodes the two old ones as $x=$ $2^{x_{1}} 3^{x_{2}}$, and then transitions in the 2-dimensional game are encoded using gadgets in which the other player may interrupt to demand proof that the required inequalities for $x_{1}$ and $x_{2}$ were satisfied. The energy functions in the so-constructed 1-dimensional automaton are piecewise affine because the original ones were integer updates. The details of the proof are in appendix.

\section{Conclusion}

We have in this paper introduced a functional framework for modeling and analyzing energy problems. We have seen that our framework encompasses most existing formal approaches to energy problems, and that it allows an application of the theory of automata over semirings and semimodules to solve reachability and Büchi acceptance problems in a generic way. For the important class of piecewise affine energy functions, we have shown that reachability and Büchi acceptance are PSPACE-hard and decidable in EXPTIME. As our algorithm is static, computations do not have to be repeated in case the initial energy changes. Also, decidability of Büchi acceptance implies that LTL model checking is decidable for energy automata.

In the last part of this paper, we have seen that one quickly comes into trouble with undecidability if the class of energy functions is extended or if twoplayer games are considered. This can be remedied by considering approximate solutions instead, using notions of distances for energy automata akin to the ones in 21] to provide quantitative measures for similar energy behavior.

Another issue that remains to be investigated is reachability and Büchi problems for one-dimensional energy automata with flat energy functions; we plan to do this in future work.

\section{References}

1. P. A. Abdulla, A. Bouajjani, and J. d'Orso. Monotonic and downward closed games. J. Log. Comput., 18(1):153-169, 2008.

2. P. A. Abdulla, K. Čerāns, B. Jonsson, and Y.-K. Tsay. Algorithmic analysis of programs with well quasi-ordered domains. Inf. Comput., 160(1-2):109-127, 2000.

3. R. Alur and D. L. Dill. A theory of timed automata. Theor. Comput. Sci., 126(2):183-235, 1994.

4. R. Alur, S. L. Torre, and G. J. Pappas. Optimal paths in weighted timed automata. In $H S C C$, pp. 49-62, 2001.

5. G. Behrmann, A. Fehnker, T. Hune, K. G. Larsen, P. Pettersson, J. Romijn, and F. W. Vaandrager. Minimum-cost reachability for priced timed automata. In HSCC, pp. 147-161, 2001.

6. S. L. Bloom and Z. Ésik. Iteration Theories: The Equational Logic of Iterative Processes. EATCS monographs on theoretical computer science. Springer, 1993. 
7. P. Bouyer, U. Fahrenberg, K. G. Larsen, and N. Markey. Timed automata with observers under energy constraints. In HSCC, pp. 61-70, 2010.

8. P. Bouyer, U. Fahrenberg, K. G. Larsen, N. Markey, and J. Srba. Infinite runs in weighted timed automata with energy constraints. In FORMATS, pp. 33-47, 2008.

9. P. Bouyer, K. G. Larsen, and N. Markey. Lower-bound constrained runs in weighted timed automata. In QEST, pp. 128-137, 2012.

10. T. Brázdil, P. Jančar, and A. Kučera. Reachability games on extended vector addition systems with states. In ICALP, pp. 478-489, 2010.

11. J. Chaloupka. Z-reachability problem for games on 2-dimensional vector addition systems with states is in P. In RP, pp. 104-119, 2010.

12. T.-h. Chan. The boundedness problem for three-dimensional vector addition systems with states. Inf. Proc. Letters, 26(6):287-289, 1988.

13. K. Chatterjee and L. Doyen. Energy parity games. In ICALP, pp. 599-610, 2010.

14. K. Chatterjee, L. Doyen, T. A. Henzinger, and J.-F. Raskin. Generalized meanpayoff and energy games. In FSTTCS, pp. 505-516, 2010.

15. J. H. Conway. Regular Algebra and Finite Machines. Chapman and Hall, 1971.

16. A. Degorre, L. Doyen, R. Gentilini, J.-F. Raskin, and S. Torunczyk. Energy and mean-payoff games with imperfect information. In CSL, pp. 260-274, 2010.

17. M. Droste, W. Kuich, and H. Vogler. Handbook of Weighted Automata. Springer, 2009 .

18. Z. Ésik and W. Kuich. An algebraic generalization of omega-regular languages. In MFCS, pp. 648-659, 2004.

19. Z. Ésik and W. Kuich. A semiring-semimodule generalization of $\omega$-regular languages, Parts 1 and 2. J. Aut. Lang. Comb., 10:203-264, 2005.

20. U. Fahrenberg, L. Juhl, K. G. Larsen, and J. Srba. Energy games in multiweighted automata. In ICTAC, pp. 95-115, 2011.

21. U. Fahrenberg, A. Legay, and C. Thrane. The quantitative linear-time-branchingtime spectrum. In FSTTCS, pp. 103-114, 2011.

22. A. Finkel and P. Schnoebelen. Well-structured transition systems everywhere! Theor. Comput. Sci., 256(1-2):63-92, 2001.

23. D. Kozen. On Kleene algebras and closed semirings. In MFCS, pp. 26-47, 1990.

24. D. Kozen. A completeness theorem for kleene algebras and the algebra of regular events. Inf. Comput., 110(2):366-390, 1994.

25. V. Mathieu and J. Desharnais. Verification of pushdown systems using omega algebra with domain. In RelMiCS, pp. 188-199, 2005.

26. D. Perrin and J.-E. Pin. Infinite Words: Automata, Semigroups, Logic and Games. Academic Press, 2004.

27. K. Quaas. On the interval-bound problem for weighted timed automata. In LATA, pp. 452-464, 2011.

28. J.-F. Raskin, M. Samuelides, and L. V. Begin. Games for counting abstractions. Electr. Notes Theor. Comput. Sci., 128(6):69-85, 2005.

29. P. Schnoebelen. Lossy counter machines decidability cheat sheet. In RP, pp. 51-75, 2010.

30. T. Wilke. An Eilenberg theorem for infinity-languages. In $I C A L P$, pp. 588-599, 1991. 


\section{Appendix: Proofs}

Proof of Lemma 1, The pointwise supremum of any set of extended energy functions is an extended energy function. Indeed, if $f_{i}, i \in I$ are extended energy functions and $x<y$ in $\mathbb{R}_{\geq 0}$, then $f_{i}(y) \geq f_{i}(x)+y-x$ for all $i$. It follows that $\sup _{i \in I} f_{i}(y) \geq \sup _{i \in I} f_{i}(x)+y-x$. Also, since $f_{i}(\perp)=\perp$ for all $i \in$ $I, \sup _{i \in I} f_{i}(\perp)=\perp$. Finally, if there is some $i$ such that $f_{i}(\infty)=\infty$, then $\sup _{i \in I} f_{i}(\infty)=\infty$. Otherwise each function $f_{i}$ is constant with value $\perp$.

The fact that $\left(\sup _{i \in I} f_{i}\right) h=\sup _{i \in I} f_{i} h$ is now clear, since the supremum is taken pointwise: For all $x,\left(\left(\sup _{i \in I} f_{i}\right) h\right)(x)=\left(\sup _{i \in I} f_{i}\right)(h(x))=\sup _{i \in I}\left(f_{i}(h(x))\right)$ and also $\left(\sup _{i \in I} f_{i} h\right)(x)=\sup _{i \in I}\left(f_{i}(h(x))\right)$.

Proof of Lemma 2. Recall first 17 that an idempotent semiring is an algebraic structure $(S, \oplus, \otimes, \nvdash, \nVdash)$ satisfying, for all $a, b, c \in S$, the following axioms:

$$
\begin{gathered}
a \oplus(b \oplus c)=(a \oplus b) \oplus c \quad a \oplus b=b \oplus a \quad a \oplus \nvdash=a \\
a \otimes(b \otimes c)=(a \otimes b) \otimes c \quad \nVdash \otimes a=a \otimes \nVdash=a \\
a \otimes(b \oplus c)=(a \otimes b) \oplus(a \otimes c) \quad \nvdash \otimes a=a \otimes \nvdash=\nvdash \\
(a \oplus b) \otimes c=(a \otimes c) \oplus(b \otimes c) \quad a \oplus a=a
\end{gathered}
$$

Now the axioms for $\vee$ in (6) are clear: maximum is associative and commutative, with neutral element \pm . Similarly, (7) states that composition is associative with neutral element the identity function id. As for (8), left distributivity of $\circ$ over $\vee$ follows from monotonicity of the functions in $\mathcal{E}$ : we have $(h(f \vee g))(x)=h(\max (f(x), g(x)))$ and $(h f \vee h g)(x)=\max (h(f(x)), h(g(x)))$. The fact that $\perp$ is absorbing is clear. Right distributivity in (9) can be similarly shown (but does not need monotonicity), and $\vee$ is idempotent by definition. $\leq$ is the natural order on $\mathcal{E}$ because $\vee$ is given pointwise.

Proof of Lemma 3, It is clear that $f^{*}$ is an energy function for any $f \in \mathcal{E}$. For the other claims, we first note that if $g \in \mathcal{E}$ is such that there is $k$ for which $g(x)=x$ for $x<k$ and $g(x)=\infty$ for $x>k$, then $g^{*}(x)=g(x)$ for $x \neq k$, and if $g(k)=k$ or $g(k)=\infty$, then also $g^{*}(k)=g(k)$.

Now let $g \in \mathcal{E}$. If there is $f \in \mathcal{E}$ with $g=f^{*}$, then we set $k=\sup \{x \mid f(x) \leq$ $x\}$. Then $f(x)>x$ and hence $g(x)=\infty$ for all $x>k$, and whenever $x<k$, then there is $y$ with $x \leq y \leq k$ and $f(y) \leq y$, hence by (*),$f(x) \leq x$, so that $g(x)=x$. If $f(k) \leq k$, then $g(k)=k$, otherwise $g(k)=\infty$ as claimed.

Proof of Lemma 4, We have $g f^{*}(\perp)=\perp=\sup _{n} g f^{n}(\perp)$, and also $g f^{*}(\infty)=$ $\infty=\sup _{n} g f^{n}(\infty)$. Also, if $g= \pm$, then $g f^{*}(x)=\perp=\sup _{n} g f^{n}(x)$ for all $x \in[0, \infty]_{\perp}$.

We are left with showing $g f^{*}(x)=\sup _{n} g f^{n}(x)$ for all $x \in \mathbb{R}_{\geq 0}$ in case $g \neq \pm$. Let thus $x \in \mathbb{R}_{>0}$. If $f(x) \leq x$, then also $f^{n}(x) \leq x$ for all $n \in \mathbb{N}$, hence $g f^{n}(x) \leq g(x)$ for all $n$, so that $\sup _{n} g f^{n}(x)=g(x)=g f^{*}(x)$.

Now assume instead that $f(x)>x$, so that $f(x)-x=M>0$. By (因) applied to $x_{1}=x$ and $x_{2}=f(x)$, we have $f^{2}(x) \geq f(x)+f(x)-x=f(x)+M$, 
hence by induction, $f^{n+1}(x) \geq f^{n}(x)+M$ for all $n \in \mathbb{N}$. Hence the sequence $\left(f^{n}(x)\right)_{n \in \mathbb{N}}$ increases without bound, so that there must be $N \in \mathbb{N}$ for which $g f^{N}(x) \neq \perp$. Again using (荬), we see that $g f^{n+1}(x) \geq g f^{n}(x)+M$ for all $n \geq N$, so that also the sequence $\left(g f^{n}(x)\right)_{n \in \mathbb{N}}$ increases without bound, whence $\sup _{n} g f^{n}(x)=\infty=g f^{*}(x)$.

Proof of Proposition 1. Let $f, g, h \in \mathcal{E}$. By Lemmas 4 and 11 $g f^{*} h=$ $\left(\sup _{n} g f^{n}\right) h=\sup _{n}\left(g f^{n} h\right)$.

Proof of Lemma 5. It is easily verified that $u(f g)=(u f) g$ and $u \circ$ id $=u$ for all $u \in \mathcal{V}$ and $f, g \in \mathcal{E}$. Moreover,

$$
\begin{aligned}
((u \vee v) f)(x) & =(u \vee v)(f(x)) \\
& =u(f(x)) \vee v(f(x)) \\
& =(u f)(x) \vee(v f)(x) \\
& =(u f \vee v f)(x)
\end{aligned}
$$

and

$$
\begin{aligned}
(u(f \vee g))(x) & =u((f \vee g)(x)) \\
& =u(f(x) \vee g(x)) \\
& =u(f(x)) \vee u(g(x))
\end{aligned}
$$

for all $u, v \in V, f, g \in \mathcal{E}$ and $x \in[0, \infty]_{\perp}$, since $u$ preserves the order. Finally, $( \pm \circ f)(x)=\mathrm{ff}= \pm(x)$ and $(u \circ \pm)(x)=\mathrm{ff}= \pm(x)$ for all $u \in \mathcal{V}, f \in \mathcal{E}$ and $x \in[0, \infty]_{\perp}$. This shows that $\mathcal{V}$ is a right $\mathcal{E}$-semimodule. The other claims follow from the embedding of $\mathcal{V}$ into $\mathcal{E}$.

Proof of Lemma 6. To show the first equality, let $x_{0} \in[0, \infty]_{\perp}$ and set $x_{n+1}=f_{n}\left(x_{n}\right)$ for $n \in \mathbb{N}$. If there is $n \in \mathbb{N}$ for which $x_{n}=\perp$, then $\left(\prod_{i=0}^{\infty} f_{i}\right)\left(x_{0}\right)=$ ff by definition, and either $f_{0}\left(x_{0}\right)=\perp$ and consequently $\left(\prod_{i=1}^{\infty} f_{i}\right)\left(f_{0}\left(x_{0}\right)\right)=\mathrm{ff}$, or $f_{0}\left(x_{0}\right) \neq \perp$, but then also $\left(\prod_{i=1}^{\infty} f_{i}\right)\left(f_{0}\left(x_{0}\right)\right)=\mathrm{ff}$. If, on the other hand, $x_{n} \neq \perp$ for all $n \in \mathbb{N}$, then $\left(\prod_{i=0}^{\infty} f_{i}\right)\left(x_{0}\right)=\mathbb{t t}$ and also $\left(\prod_{i=1}^{\infty} f_{i}\right)\left(f_{0}\left(x_{0}\right)\right)=\mathbb{t}$. The second equality can be shown by similarly easy arguments.

Proof of Proposition 2, We need two technical lemmas in this proof.

Lemma 8. For all $u \in \mathcal{V}, n \in \mathbb{N}$, and $f_{1}, \ldots, f_{n+1}, g_{1}, \ldots, g_{n} \in \mathcal{E}$, we have $u f_{n+1} g_{n}^{*} f_{n} \cdots g_{1}^{*} f_{1}=\sup _{k_{1}, \ldots, k_{n} \in \mathbb{N}} u f_{n+1} g_{n}^{k_{n}} f_{n} \cdots g_{1}^{k_{1}} f_{1}$.

Proof. By induction on $n$. For $n=0$ our claim is clear. Suppose now that $n>0$ and that the claim holds for all $m<n$. Then

$$
\begin{aligned}
v f_{n+1} g_{n}^{*} \cdots g_{1}^{*} f_{1} & =\left(\sup \left\{v f_{n+1} g_{n}^{k_{n}} \cdots g_{2}^{k_{2}} f_{2} \mid k_{2}, \ldots, k_{n} \in \mathbb{N}\right\}\right) g_{1}^{*} f_{1} \\
& =\sup \left\{v f_{n+1} g_{n}^{k_{n}} \cdots g_{2}^{k_{2}} f_{2} g_{1}^{*} \mid k_{2}, \ldots, k_{n} \in \mathbb{N}\right\} f_{1} \\
& =\sup \left\{\sup \left\{v f_{n+1} g_{n}^{k_{n}} \cdots g_{2}^{k_{2}} f_{2} g_{1}^{k_{1}} \mid k_{1} \geq 0\right\} \mid k_{2}, \ldots, k_{n} \in \mathbb{N}\right\} f_{1} \\
& =\sup \left\{v f_{n+1} g_{n}^{k_{n}} \cdots g_{2}^{k_{2}} f_{2} g_{1}^{k_{1}} \mid k_{1}, \ldots, k_{n} \in \mathbb{N}\right\} f_{1} \\
& =\sup \left\{v f_{n+1} g_{n}^{k_{n}} \cdots g_{1}^{k_{1}} f_{1} \mid k_{1}, \ldots, k_{n} \in \mathbb{N}\right\} .
\end{aligned}
$$


Here the first equality is by induction hypothesis, the second by Lemma 1, the third by Lemma 4 and the fifth again by Lemma 1.

Lemma 9. For all $f, g \in \mathcal{E},(f \vee g)^{\omega}=\sup _{h_{0}, h_{1}, \ldots \in\{f, g\}} \prod_{i=0}^{\infty} h_{i}$ and $\left(g f^{*}\right)^{\omega}=$ $\sup _{k_{0}, k_{1}, \ldots \in \mathbb{N}} \prod_{i=0}^{\infty}\left(g f^{k_{i}}\right)$.

Proof. To show the first claim, note first that as infinite product preserves the order, we have $\sup _{h_{i} \in\{f, g\}} \prod_{i=0}^{\infty} h_{i} \leq(f \vee g)^{\omega}$. To complete the proof, we have to show that whenever $(f \vee g)^{\omega}(x)=\mathbb{t t}$ for some $x$, then $\left.\sup _{h_{i} \in\{f, g\}} \prod_{i=0}^{\infty} h_{i}\right)(x)=$ tt. So suppose that $(f \vee g)^{\omega}(x)=\mathbb{t t}$. Then we must have $(f \vee g)(x) \geq x$ and $x \neq \perp$, so that either $f(x) \geq x$ or $g(x) \geq x$. Without loss of generality we can assume $f(x) \geq x$, but then $f^{\omega}(x)=\mathbb{t}$ and $\left(\sup _{h_{i} \in\{f, g\}} \prod_{i=0}^{\infty} h_{i}\right)(x) \geq f^{\omega}(x)=\mathbb{t t}$.

For the second claim, it suffices to prove that if for some $x,\left(g f^{*}\right)^{\omega}(x)=\mathbb{t t}$, then there is a sequence $k_{0}, k_{1}, \ldots$ such that $\left(\prod_{i=0}^{\infty}\left(g f^{k_{i}}\right)\right)(x)=\mathbf{t t}$. Suppose that $\left(g f^{*}\right)^{\omega}(x)=\mathrm{tt}$. Then $x \neq \perp$ and $g f^{*}(x) \geq x$. We know that $g f^{*}(x)=$ $\sup _{n} g f^{n}(x)$. Thus $\sup _{n} g f^{n}(x) \geq x$. If $f(x) \leq x$, then the sequence $\left(g f^{n}(x)\right)_{n}$ is decreasing, so that $\sup _{n} g f^{n}(x)=g(x) \geq x$. We thus have $g^{\omega}(x)=\mathbb{t}$, and $\left(\prod_{i=0}^{\infty}\left(g f^{k_{i}}\right)\right)(x)=\mathbb{t t}$ holds when $k_{i}=0$ for all $i$. If, on the other hand, $f(x)>x$, then the sequence $\left(f^{n}(x)\right)_{n}$ is strictly increasing with $\sup _{n} f^{n}(x)=\infty$. Since clearly $g \neq \pm$, it follows that there is some $n$ with $g f^{n}(x) \geq x$. It follows now that $\left(g f^{n}\right)^{\omega}=\left(\prod_{i=0}^{\infty}\left(g f^{n}\right)\right)(x) \geq x$.

Now for the proof of the proposition, we show first that $(g f)^{\omega}=(f g)^{\omega} f$ for all $f, g \in \mathcal{E}$. Let $f, g \in \mathcal{E}$ and $x \in[0, \infty]_{\perp}$. The result is clear for $x=\perp$, so let $x \neq \perp$. Assume first that $(g f)(x) \geq x$, then $(g f)^{\omega}(x)=\mathbb{t t}$. But by monotonicity, also $(f g)(f(x))=f(g f)(x) \geq f(x)$, hence $\left((f g)^{\omega} f\right)(x)=\mathbf{t t}$. Now assume that, instead, $(g f)(x)<x$, then $(g f)^{\omega}(x)=\mathrm{ff}$. By (図), also $(f g)(f(x))<f(x)$, so that $\left((f g)^{\omega} f\right)(x)=\mathrm{ff}$.

Next we show that $(f \vee g)^{\omega}=f^{\omega}\left(g f^{*}\right)^{*} \vee\left(g f^{*}\right)^{\omega}$. We have

$$
\begin{aligned}
f^{\omega}\left(g f^{*}\right)^{*} & =\sup _{n} f^{\omega}\left(g f^{*}\right)^{n} \\
& =\sup _{n} \sup _{k_{n}} \cdots \sup _{k_{1}} f^{\omega} g f^{k_{n}} \cdots g f^{k_{1}} \\
& =\sup _{n, k_{1}, \ldots, k_{n}}\left(\cdots f f g f^{k_{n}} \cdots g f^{k_{1}}\right)
\end{aligned}
$$

and

$$
\left(g f^{*}\right)^{\omega}=\sup _{k_{1}, k_{2}, \ldots}\left(\cdots g f^{k_{2}} g f^{k_{1}}\right) .
$$

Using these equalities, we conclude that

$$
\begin{aligned}
f^{\omega}\left(g f^{*}\right)^{*} \vee\left(g f^{*}\right)^{\omega} & =\sup _{n, k_{1}, \ldots, k_{n}}\left(\cdots f f g f^{k_{n}} \cdots g f^{k_{1}}\right) \vee \sup _{k_{1}, k_{2}, \ldots}\left(\cdots g f^{k_{2}} g f^{k_{1}}\right) \\
& =\sup _{h_{i} \in\{f, g\}}\left(\cdots h_{2} h_{1}\right) \\
& =(f \vee g)^{\omega} .
\end{aligned}
$$


Proof of Lemma 7, We define

$$
T^{\prime}=\left\{\left(s, \sup \left\{f \mid\left(s, f, s^{\prime}\right) \in T\right\}, s^{\prime}\right) \mid s, s^{\prime} \in S\right\} ;
$$

note that $\sup \emptyset= \pm$. The idea of the construction is that if two transitions $\left(s, f, s^{\prime}\right),\left(s, f^{\prime}, s^{\prime}\right) \in T$ are available, then it does not change the automaton's behavior if we replace them by a transition labeled with $f \vee f^{\prime}$.

To see this, we note that the semantic graph of $\left(S, T^{\prime}\right)$ is above the one of $(S, T)$ in the following sense: If $(s, x) \stackrel{g}{\rightarrow}\left(s^{\prime}, x^{\prime}\right)$, with $x, x^{\prime} \neq \perp$, is a transition in the semantic graph of $\left(S, T^{\prime}\right)$, then

$$
\begin{aligned}
x^{\prime} & =\left(\sup \left\{f \mid\left(s, f, s^{\prime}\right) \in T\right\}\right)(x) \\
& =\sup \left\{f(x) \mid\left(s, f, s^{\prime}\right) \in T\right\},
\end{aligned}
$$

hence there is also a transition $(s, x) \stackrel{f}{\rightarrow}\left(s^{\prime}, x^{\prime}\right)$ in the semantic graph of $(S, T)$. On the other hand, if $(s, x) \stackrel{f}{\rightarrow}\left(s^{\prime}, x^{\prime}\right)$ is a transition in the semantic graph of $(S, T)$, then we have a transition $s \stackrel{g}{\rightarrow} s^{\prime}$ in $\left(S, T^{\prime}\right)$ with $g \geq f$, hence a transition $(s, x) \stackrel{g}{\rightarrow}\left(s^{\prime}, x^{\prime \prime}\right)$ in the semantic graph of $\left(S, T^{\prime}\right)$ with $x^{\prime \prime} \geq x^{\prime}$.

It is now clear that any accepting run (in the reachability or in the Büchi sense) in $\left(S, T^{\prime}\right)$ is also available in $(S, T)$; likewise, any accepting run in $(S, T)$ has one which is above it in $\left(S, T^{\prime}\right)$.

Proof of Theorem 3. The statement in the theorem is equivalent to asserting that there is an accepting state $j$ which is reachable from $\left(s_{0}, x_{0}\right)$ with output energy $x={ }_{\mathrm{t}} I^{j} T^{*} I^{s_{0}}\left(x_{0}\right)$, and at which there is a non-trivial loop which does not lose energy, i.e. ${ }_{\mathrm{t}} I^{j} T T^{*} I^{j}(x) \geq x$.

We shall need a few technical lemmas in the proof. For a global state $q=$ $(s, x)$, we write $\operatorname{state}(q)=s$ and $\operatorname{val}(q)=x$ below.

Lemma 10. Let $q_{0} \stackrel{f_{1}}{\longrightarrow} q_{1} \stackrel{f_{2}}{\longrightarrow} \cdots \stackrel{f_{n}}{\longrightarrow} q_{n}$ be a run of $(S, T)$ such that there are $i<j$ for which $q_{i} \rightsquigarrow q_{j}$ is a loop with $\operatorname{val}\left(q_{i}\right) \geq \operatorname{val}\left(q_{j}\right)$. Then there are global states $q_{j+1}^{\prime}, \ldots, q_{n}^{\prime}$ with state $\left(q_{k}^{\prime}\right)=\operatorname{state}\left(q_{k}\right)$ for all $k$, and such that

$$
q_{0} \stackrel{f_{1}}{\longrightarrow} q_{1} \stackrel{f_{2}}{\longrightarrow} \cdots \stackrel{f_{i}}{\longrightarrow} q_{i} \stackrel{f_{j+1}}{\longrightarrow} q_{j+1}^{\prime} \stackrel{f_{j+2}}{\longrightarrow} \cdots \stackrel{f_{n}}{\longrightarrow} q_{n}^{\prime}
$$

is a run of $(S, T)$ with $\operatorname{val}\left(q_{n}^{\prime}\right) \geq \operatorname{val}\left(q_{n}\right)$.

Proof. By val $\left(q_{i}\right) \geq \operatorname{val}\left(q_{j}\right)$ and (因), also val $\left(q_{j+1}^{\prime}\right) \geq \operatorname{val}\left(q_{j+1}\right)$. Inductive appli-

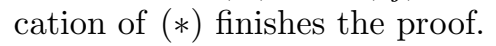

Lemma 11. Let $q_{0} \stackrel{f_{1}}{\longrightarrow} q_{1} \stackrel{f_{2}}{\longrightarrow} \cdots \stackrel{f_{n}}{\longrightarrow} q_{n}$ be a run of $(S, T)$ such that there are $i<j$ for which $q_{i} \rightsquigarrow q_{j}$ is a loop with $\operatorname{val}\left(q_{j}\right)>\operatorname{val}\left(q_{i}\right)$. Then there are $\Delta>0$ and, for each $k \geq 1$, global states $q_{i+1}^{l}, \ldots, q_{j}^{l}$ for all $1 \leq l \leq k$, and $q_{j+1}^{k}, \ldots, q_{n}^{k}$, with $\operatorname{state}\left(q_{m}^{l}\right)=\operatorname{state}\left(q_{m}\right)$ for each $k, l$, such that

$$
q_{0} \stackrel{f_{1}}{\longrightarrow} \cdots \stackrel{f_{i}}{\longrightarrow} q_{i}\left(\stackrel{f_{i+1}}{\longrightarrow} q_{i+1}^{l} \stackrel{f_{i+2}}{\longrightarrow} \cdots \stackrel{f_{j}}{\longrightarrow} q_{j}^{l}\right)_{1 \leq l \leq k} \stackrel{f_{j+1}}{\longrightarrow} q_{j+1}^{k} \stackrel{f_{j+2}}{\longrightarrow} \cdots \stackrel{f_{n}}{\longrightarrow} q_{n}^{k}
$$

is a run of $(S, T)$, and $\operatorname{val}\left(q_{n}^{k+1}\right) \geq \operatorname{val}\left(q_{n}^{k}\right)+\Delta$. 
Proof. Let $\Delta=\operatorname{val}\left(q_{j}\right)-\operatorname{val}\left(q_{i}\right)>0$. By (因), $\operatorname{val}\left(q_{j}^{l+1}\right)-\operatorname{val}\left(q_{j}^{l}\right) \geq \Delta$ for all $l$, hence also $\operatorname{val}\left(q_{n}^{k+1}\right)-\operatorname{val}\left(q_{n}^{k}\right) \geq \Delta$.

We say that a loop $q_{i} \rightsquigarrow q_{j}$ with $\operatorname{val}\left(q_{j}\right)>\operatorname{val}\left(q_{i}\right)$ as in Lemma 11 is energy producing; similarly, a loop with val $\left(q_{j}\right)<\operatorname{val}\left(q_{i}\right)$ will be called energy consuming. As a corollary of the last lemma, the energy value at state $\left(q_{n}\right)$ can be pushed arbitrarily high: for any $M \in \mathbb{R}_{>0}$, there exists $k$ such that the $k$-iteration $q_{0} \rightsquigarrow q_{i}\left(\rightarrow q_{i+1}^{l} \rightarrow \cdots \rightarrow q_{j}^{l}\right)_{1 \leq 1 \leq k} \rightarrow q_{j+1}^{k} \rightsquigarrow q_{n}^{k}$ has val $\left(q_{n}^{k}\right) \geq M$. We also remark that similar results are available for infinite runs; also with these we can remove loops which are not energy producing and iterate loops which are.

Lemma 12. Let $f \in \mathcal{E}$ and $x \in \mathbb{R}_{\geq 0}$. If $f(x)<x$, then $\lim _{n \rightarrow \infty} f^{n}(x)=\perp$.

Proof. We have $x-f(x)=M>0$. Using (函), we see that $f^{n+1}(x) \leq f^{n}(x)-M$ for all $n \in \mathbb{N}$. Hence $\left(f^{n}(x)\right)_{n \in \mathbb{N}}$ decreases without bound, so that there must be $N \in \mathbb{N}$ such that $f^{n}(x)=\perp$ for all $n \geq N$.

Now for the proof of the theorem, the backwards direction is clear, as the cycle at $j$ contains an accepting state and can be iterated indefinitely because of ${ }_{\mathrm{t}} I^{j} T T^{*} I^{j}(x) \geq x$. For the forward direction, let $\rho$ be an infinite run from $\left(s_{0}, x_{0}\right)$ which visits $F$ infinitely often, then $\rho$ must contain an accepting state $s \leq k$ infinitely often, so we can write

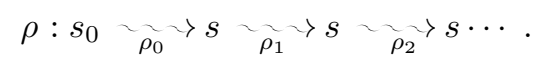

Now inductively for each $i \geq 1$, we modify $\rho$ as follows:

- If $\rho_{i}$ is not energy consuming, we can use Lemma 11] to replace $\rho$ by the run $\rho_{0} \cdots \rho_{i} \rho_{i}^{1} \rho_{i}^{2} \cdots$, where each $\rho_{i}^{j}$ is a loop over the same cycle as $\rho_{i}$. We have constructed a run which consists of a finite prefix and a loop.

- In case $\rho_{i}=\mu_{1} \mu_{2} \mu_{3}$ strictly contains a loop $\mu_{2}$ which is energy producing, we can use Lemma 11 to iterate $\mu_{2}$ sufficiently often so that the combined run $\tilde{\rho}_{i}=\mu_{1} \mu_{2} \mu_{2}^{1} \cdots \mu_{2}^{N} \mu_{3}^{\prime}$, where each $\mu_{2}^{j}$ is a loop over the same cycle as $\mu_{2}$ and $\mu_{3}^{\prime}$ over the same path as $\mu_{3}$, is not energy consuming. Using Lemma11 again, we can now replace $\rho$ by the run $\rho_{0} \cdots \rho_{i-1} \tilde{\rho}_{i} \tilde{\rho}_{i}^{1} \tilde{\rho}_{i}^{2} \cdots$, where each $\tilde{\rho}_{i}^{j}$ is a loop over the same cycle as $\tilde{\rho}_{i}$. This run consists of a finite prefix and a loop.

- Any loops properly contained in $\rho_{i}$ which are not energy producing can be removed by Lemma 10 .

Assume, for the sake of contradiction, that the above induction never finishes. Then we have constructed a run $\rho^{\prime}=\rho_{0} \rho_{1}^{\prime} \rho_{2}^{\prime} \ldots$ in which each $\rho_{i}^{\prime}$ is an energy consuming simple loop from $s$ to $s$. There are only finitely many simple cycles from $s$ to $s$, thus one of them appears infinitely often as a cycle underlying a simple loop in $\rho^{\prime}$. Call this cycle $\pi$ and let $\sigma_{1}, \sigma_{2}, \ldots$ be the loops in $\rho^{\prime}$ over it.

We can hence write $\rho^{\prime}=\rho_{0} \mu_{1} \sigma_{1} \mu_{2} \sigma_{2} \cdots$. All loops $\mu_{i}$ are energy consuming, hence can be removed from $\rho^{\prime}$ by Lemma 10. We have constructed an infinite 


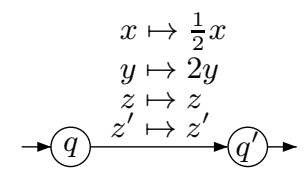

Fig. 6. Module for $\left(q, c_{1}++, q^{\prime}\right)$

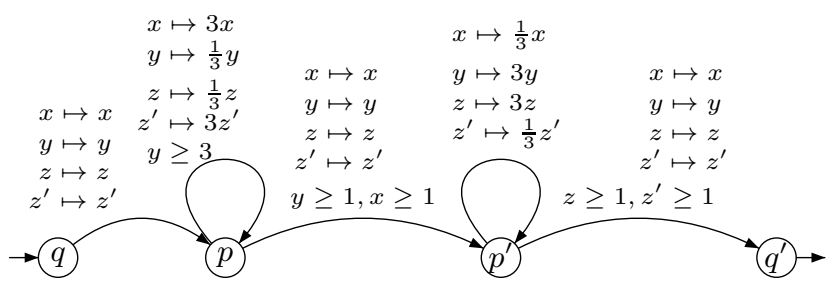

Fig. 7. Module for $\left(q, c_{1}=0 ?, q^{\prime}\right)$

run $\rho_{0} \sigma_{1}^{\prime} \sigma_{2}^{\prime} \cdots$ in $(S, T)$ in which each $\sigma_{i}^{\prime}$ is energy consuming. The sequence of energy values after each iteration $\sigma_{i}^{\prime}$ is given by $\left(f_{\pi}^{n}\left(f_{\rho_{0}}\left(x_{0}\right)\right)\right)_{n \in \mathbb{N}}$, but by Lemma 12 and as $f_{\pi}\left(f_{\rho_{0}}\left(x_{0}\right)\right)<f_{\rho_{0}}\left(x_{0}\right)\left(f_{\pi}\left(f_{\rho_{0}}\left(x_{0}\right)\right)\right.$ is the energy value after the first loop $\left.\sigma_{1}^{\prime}\right), \lim _{n \rightarrow \infty} f_{\pi}^{n}\left(f_{\rho_{0}}\left(x_{0}\right)\right)=f_{\pi}^{\omega}\left(f_{\rho_{0}}\left(x_{0}\right)\right)=\perp$, a contradiction.

Proof of Theorem 7. We reduce from the halting problem for 2-counter machines. We use two energy variables $x, y$ to encode the values of the counters $c_{1}$ and $c_{2}$, and we use two additional energy variables $z, z^{\prime}$ for storing temporary information needed for encoding zero tests and decrementation operations of the 2 -counter machine. The initial value of all energy variables is 1 . Let $\left(q, c_{1}{ }^{++}, q^{\prime}\right)$ be a transition of the 2-counter machine that increments the value of the first counter. The module simulating this transition is shown in Fig. 6. The intuition is that $x$ and $y$ encode the counter values as $x=1 /\left(2^{c_{1}} 3^{c_{2}}\right)$ and $y=2^{c_{1}} 3^{c_{2}}$, respectively. Hence $x$ is divided and $y$ is multiplied by 2 to encode the incrementation of $c_{1}$. Likewise, an incrementation of $c_{2}$ is encoded by dividing $x$ and multiplying $y$ by 3 . Let $\left(q, c_{1}=0\right.$ ?, $\left.q^{\prime}\right)$ be a zero test transition for $c_{1}$. The corresponding module is shown in Fig. 7. Note that in order to take the transition from $p$ to $p^{\prime}$, the values of both $x$ and $y$ have to be greater than or equal to 1 . This is the case if and only if we loop in $p$ exactly $c_{2}$ times and $c_{1}=0$. In $p^{\prime}$ we have to loop for the same number of times as in $p$ to restore the original values of $x$ and $y$. For this we use the information stored in $z$ and $z^{\prime}$ together with the lower bound restrictions at the transition from $p^{\prime}$ to $q^{\prime}$. For the simulation of zero test transitions for $c_{2}$, we replace $\frac{1}{3}$ and 3 by $\frac{1}{2}$ and 2 , respectively. The idea for encoding a decrementation operation of $c_{1}$ and $c_{2}$ is similar.

Proof of Theorem [9. We show a reduction from reachability games on 2dimensional $\mathcal{E}_{\text {int }}$-automata to reachability games on 1-dimensional $\overline{\mathcal{E}}_{\text {pw }}$-automata.

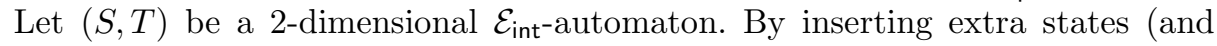
transitions) if necessary, we can assume that for any $\left(s,(f, g), s^{\prime}\right) \in T$, either 

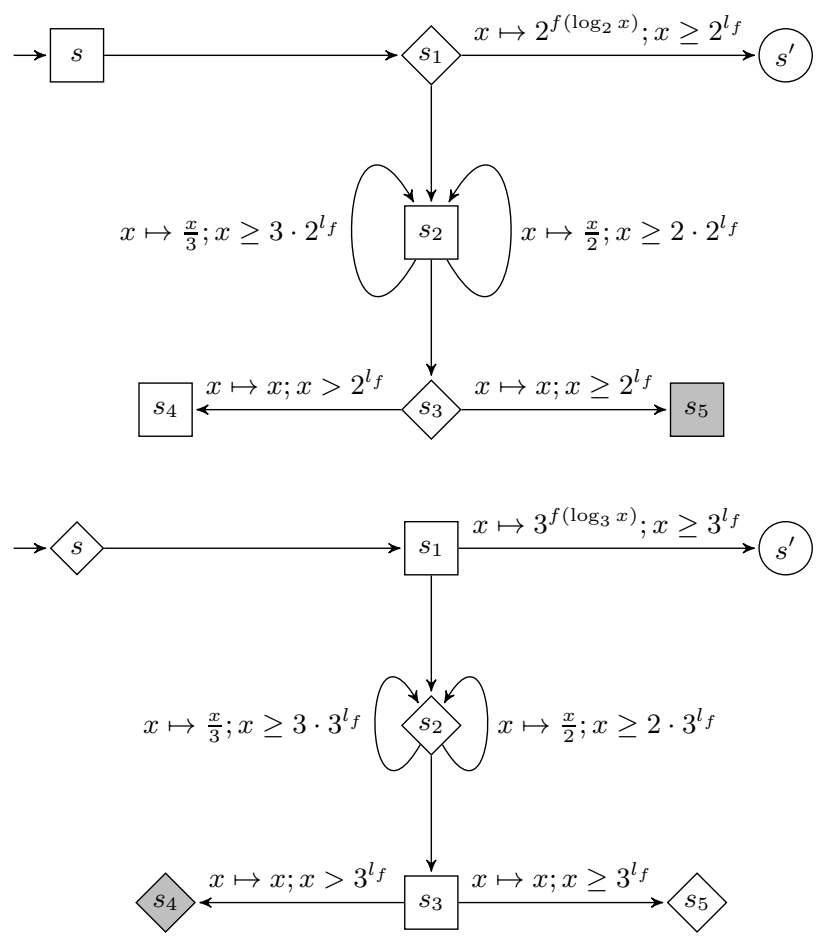

Fig. 8. Conversion of two types of edges in $(S, T)$. Top: an edge $\left(s,(f\right.$, id $\left.), s^{\prime}\right)$ from a player- $A$ state $s$; bottom: an edge $\left(s,(\right.$ id, $\left.f), s^{\prime}\right)$ from a player- $B$ state $s$. Player- $A$ states are depicted using squares, player- $B$ states are diamonds. Accepting states have a gray background color. The ownership of state $s^{\prime}$ is unchanged.

$f=$ id with $l_{f}=0$, or $g=$ id with $l_{g}=0$. We build an energy automaton $\left(S^{\prime}, T^{\prime}\right)$.

Let $\left(s,\left(f\right.\right.$, id), $\left.s^{\prime}\right) \in T$ be a player- $A$ transition (i.e. $\left.s \in S_{A}\right)$ in $(S, T)$ (with lower bound $l_{f}$ as usual), then we model this in $\left(S^{\prime}, T^{\prime}\right)$ using $s, s^{\prime}$ and the following new states and transitions; see Figure 8 for a pictorial description.

- player- $A$ states: $s_{2}, s_{4}, s_{5}$ (accepting); player- $B$ states: $s_{1}, s_{3}$

- transitions:

- $\left(s,[x \mapsto x ; x \geq 0], s_{1}\right) ;\left(s_{1},\left[x \mapsto 2^{f\left(\log _{2} x\right)} ; x \geq 2^{l_{f}}\right], s^{\prime}\right)$

- $\left(s_{1},[x \mapsto x ; x \geq 0], s_{2}\right)$

- $\left(s_{2},\left[x \mapsto \frac{x}{3} ; x \geq 3 \cdot 2^{l_{f}}\right], s_{2}\right) ;\left(s_{2},\left[x \mapsto \frac{x}{2} ; x \geq 2 \cdot 2^{l_{f}}\right], s_{2}\right)$

- $\left(s_{2},[x \mapsto x ; x \geq 0], s_{3}\right)$

- $\left(s_{3},\left[x \mapsto x ; x>2^{l_{f}}\right], s_{4}\right) ;\left(s_{3},\left[x \mapsto x ; x \geq 2^{l_{f}}\right], s_{5}\right)$

Note that $s_{4}$ is a deadlock state, hence player $A$ loses the reachability game if $s_{4}$ is reached. Similarly, she wins if $s_{5}$ is reached.

The intuition is that the new energy variable $x$ encodes the two old ones as $x=2^{x_{1}} 3^{x_{2}}$. If player $A$ wants to bring $\left(S^{\prime}, T^{\prime}\right)$ from $s$ to $s^{\prime}$, and commits to this 
by taking the transition $s \rightarrow s_{1}$, she may be interrupted by player $B$ taking the $s_{1} \rightarrow s_{2}$ transition. Here player $A$ has to prove that $x_{1}$ was really $\geq l_{f}$, by using the loops at $s_{2}$ to bring $x$ to the precise value $2^{l_{f}}$. If she manages this, then player $B$ has only the $s_{3} \rightarrow s_{5}$ transition available in $s_{3}$, hence player $A$ wins. Otherwise, player $B$ wins.

The conversions of other types of transitions are similar. One can easily see that player $A$ can reach a state in $F$ in the original energy automaton $(S, T)$ if, and only if, she can reach a state in $F$, or one of the new accepting states, in the new automaton $\left(S^{\prime}, T^{\prime}\right)$.

We miss to argue that all energy functions in $\left(S^{\prime}, T^{\prime}\right)$ are piecewise affine. Looking at the defined modules, we see that this is the case except perhaps for the functions defined as $g_{2}(x)=2^{f\left(\log _{2} x\right)}$ and $g_{3}(x)=3^{f\left(\log _{3} x\right)}$. However, $f$ is an integer update function, so that $f(x)=x+k$ for some $k \in \mathbb{Z}$; hence $g_{2}(x)=2^{k} x$ and $g_{3}(x)=3^{k} x$, which are indeed piecewise affine. 